\title{
Effects of the Interstellar Medium on Detection of Low-frequency Gravitational Waves
}

\author{
Dan Stinebring \\ Oberlin College, Dept. of Physics and Astronomy, Oberlin, OH 44074 \\ E-mail: dan.stinebring@oberlin.edu
}

\begin{abstract}
Time variable delays due to radio wave propagation in the ionized interstellar medium are a substantial source of error in pulsar timing array efforts. We describe the physical origin of these effects, discussing dispersive and scattering effects separately. Where possible, we give estimates of the magnitude of timing errors produced by these effects and their scaling with radio frequency. Although there is general understanding of the interstellar medium propagation errors to be expected with pulsar timing array observations, detailed comparison between theory and practice is still in its infancy, particularly with regard to scattering effects.
\end{abstract}

\section{Introduction}

As is clear from other articles in this volume, the success of the pulsar timing array (PTA) effort depends on measuring pulse arrival times to an accuracy of tens of nanoseconds over five years or more. Since the radio waves from pulsars travel through hundreds or thousands of light years of interstellar space before arriving at our telescopes, we need to accurately correct for timing delays caused by this passage. It is the ionized component of the interstellar medium (ISM) that interacts strongly with radio waves and causes a myriad of effects that are primarily deleterious to pulsar timing.

The two broad classes of propagation effects are those due to dispersion (figure 1), which would be present in a homogeneous medium and scattering, which is due to spatial inhomogeneities in the medium. All effects are traceable to the oscillations of the plasma induced by the passage of the e-m wave of frequency $\nu$. Dispersion arises when the radio waves, which span a range of frequencies, interacts with the plasma. The frequency-dependent group velocity is $v_{g}=c \sqrt{1-\nu_{p}^{2} / \nu^{2}}$, where the plasma frequency is $\nu_{p}=\sqrt{n_{e} e^{2} /\left(\pi m_{e}\right)} \approx\left(10^{4} \mathrm{~Hz}\right) \sqrt{n_{e} / 1 \mathrm{~cm}^{-3}}$, with the electron density $n_{e} \approx 0.03 \mathrm{~cm}^{-3}$ in the Galactic plane. When the radio wave propagates through a column of free electrons characterized by the dispersion measure $\mathrm{DM}=\int n_{e} d s$, it will be delayed by a time equal to [1]

$$
t_{\mathrm{DM}}(\nu)=\frac{e^{2}}{2 \pi m_{e} c^{3} \nu^{2}} \mathrm{DM} \approx(4.15 \mathrm{~ms}) \frac{\mathrm{DM}}{\nu_{\mathrm{GHz}}^{2}},
$$




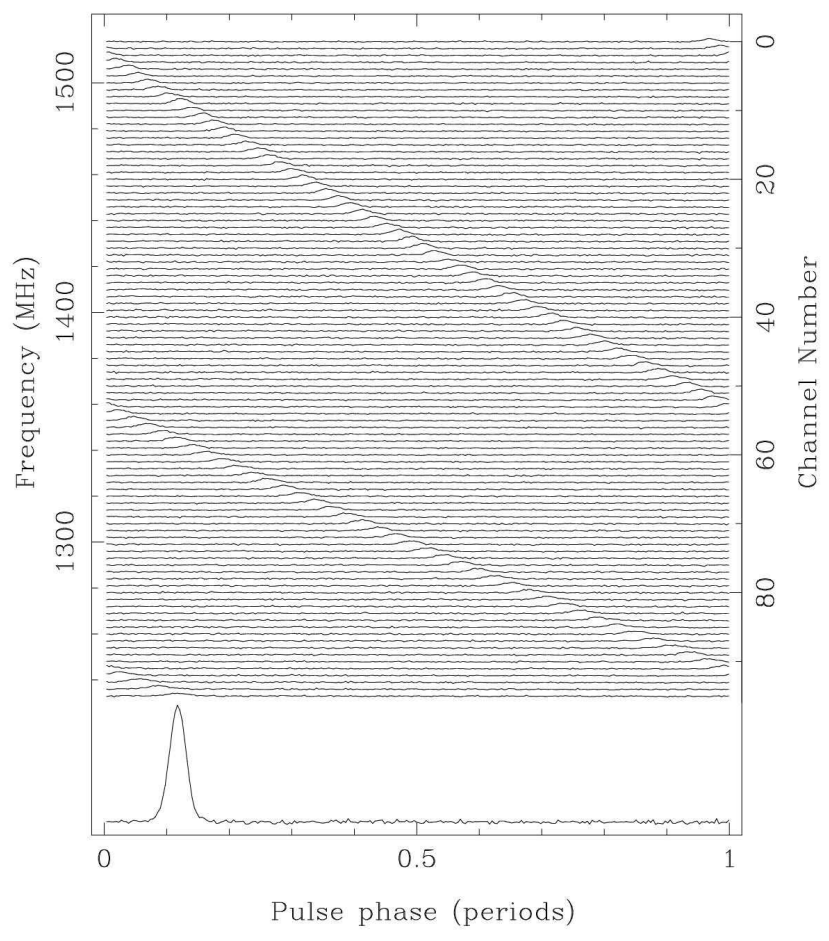

Figure 1. Dispersive delay for a pulsar observation centered at $1380 \mathrm{MHz}$. Over this fairly wide fractional bandwidth the dispersive slope changes significantly. Pulses are folded modulo the pulsar period causing the apparent discontinuity around channel 52.From [1]

where, in the last expression, DM is in the standard units of $\mathrm{pc} \mathrm{cm}^{-3}$.

A homogeneous ionized ISM would produce dispersion; in fact, an ionized medium that has variations in $n_{e}$ only along the propagation direction produces dispersive effects that are completely correctable. However, spatial inhomogeneities transverse to the line of sight produce multi-path propagation effects or scattering. There are multiple effects but the most important for this discussion are intensity scintillations and pulse broadening.

If pulsars, the Earth, and the interstellar medium were stationary the constant time delay produced by both dispersion and scattering would present no problem for PTA efforts. However, pulsars are high velocity objects, in general [1, and the Earth and ISM motions cannot be neglected in all cases. This motion gives rise to variable delays in the time of arrival (TOA) of pulses which, if uncorrected or improperly corrected, produce systematic errors that can swamp any GW signal. Figure 2 shows examples of time variable dispersion measure and time variable scattering delay - including occasional rapid fluctuations (timescales of days) - that require careful detection and mitigation. 

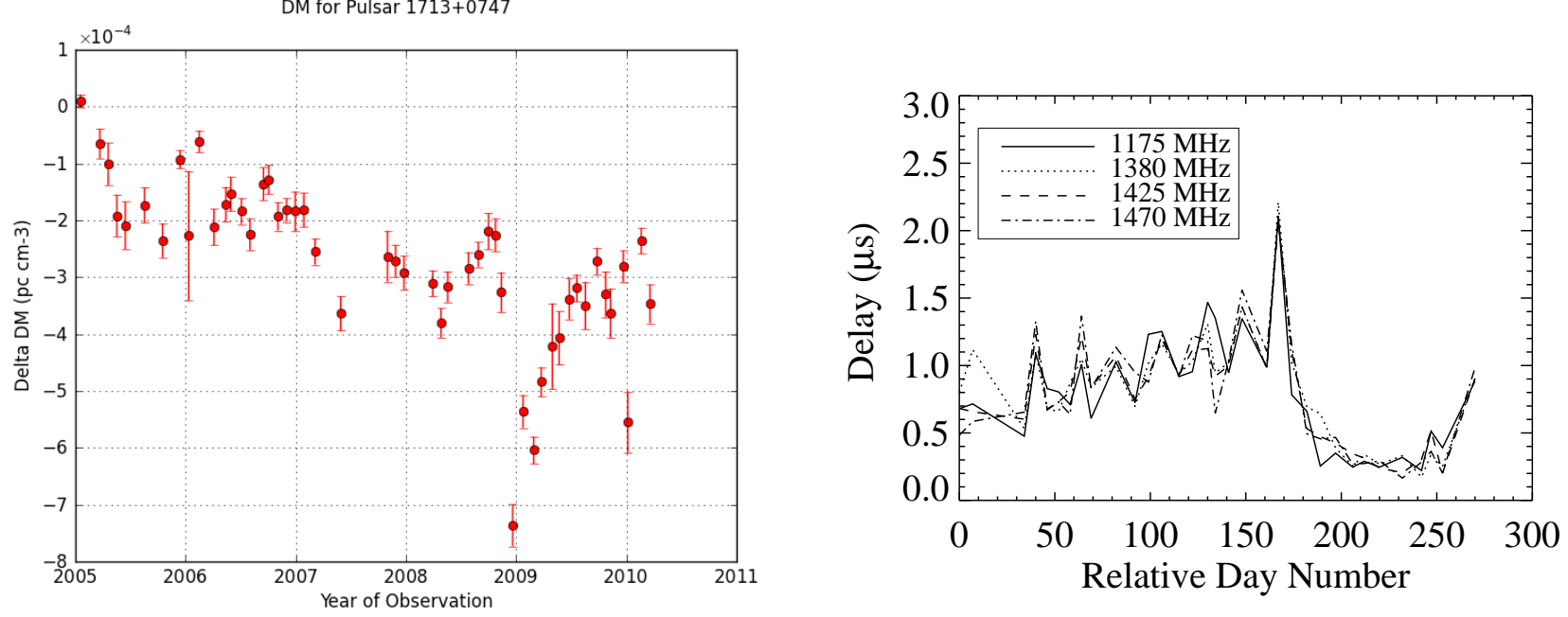

Figure 2. (Left) Dispersion measure variations of PSR J1713+0747 for approximately five years from dual-frequency NANOGrav observations 2. A fluctuation of DM by $1.0 \times 10^{-4} \mathrm{pc} \mathrm{cm}^{-3}$ produces an arrival time variation of $0.41 \mu \mathrm{s}$ at an observing frequency of $1 \mathrm{GHz}$. The abrupt dip and recovery near the start of 2009 was corroborated by other PTAs $[3$, which also monitor this pulsar. (Right) Scattering delay from PSR B1713+37 as determined indirectly from the power distribution in the secondary spectra 4. The four curves have been shifted vertically using $1380 \mathrm{MHz}$ as a reference frequency.

\section{The ionized interstellar medium}

Before proceeding we set the basic scenario and define some important quantities. In figure 3 we see the effects of multi-path scattering and the ensuing interference because of the very compact size of pulsars as radio sources. Both a dynamic spectrum and its $2 \mathrm{~d}$ power spectrum (the "secondary spectrum") are shown. The dynamic spectrum is comprised of scintles, which are isolated islands of power in the frequency-time plane. They arise in the time dimension because the random interference pattern at the location of the Earth has a characteristic spatial scale and this becomes a characteristic time scale due to the relative motion in the problem. The scintles have a characteristic extent in frequency because the coherent rays arriving at the Earth have a range of extra scattering delays of $\sim \tau$ and the uncertainty principle gives rise to a diffractive bandwidth $\Delta \nu_{d} \sim(2 \pi \tau)^{-1}$. See [5] for a careful definition of all quantities and a discussion of how they depend on the spectrum of density inhomogeneities in the medium and the spatial distribution of scattering material along the line of sight. It is often valid to assume a "frozen flow" approximation since pulsar transverse velocities are typically $>100 \mathrm{~km} / \mathrm{s}$, the Earth's contribution is $\sim 30 \mathrm{~km} / \mathrm{s}$, whereas the thermal speed of the ISM is $\sim 10 \mathrm{~km} / \mathrm{s}$.

From secondary spectra similar to this [6, 7, 8, 19, 10, 11, 12, 13, 14, 15, there is evidence that the majority of scattering along many lines of sight to nearby pulsars takes place in discrete screens, by which we mean a relatively small fraction (upper limits $\lesssim$ a few percent) of the line of sight (LOS) distance. This simplifies a description 

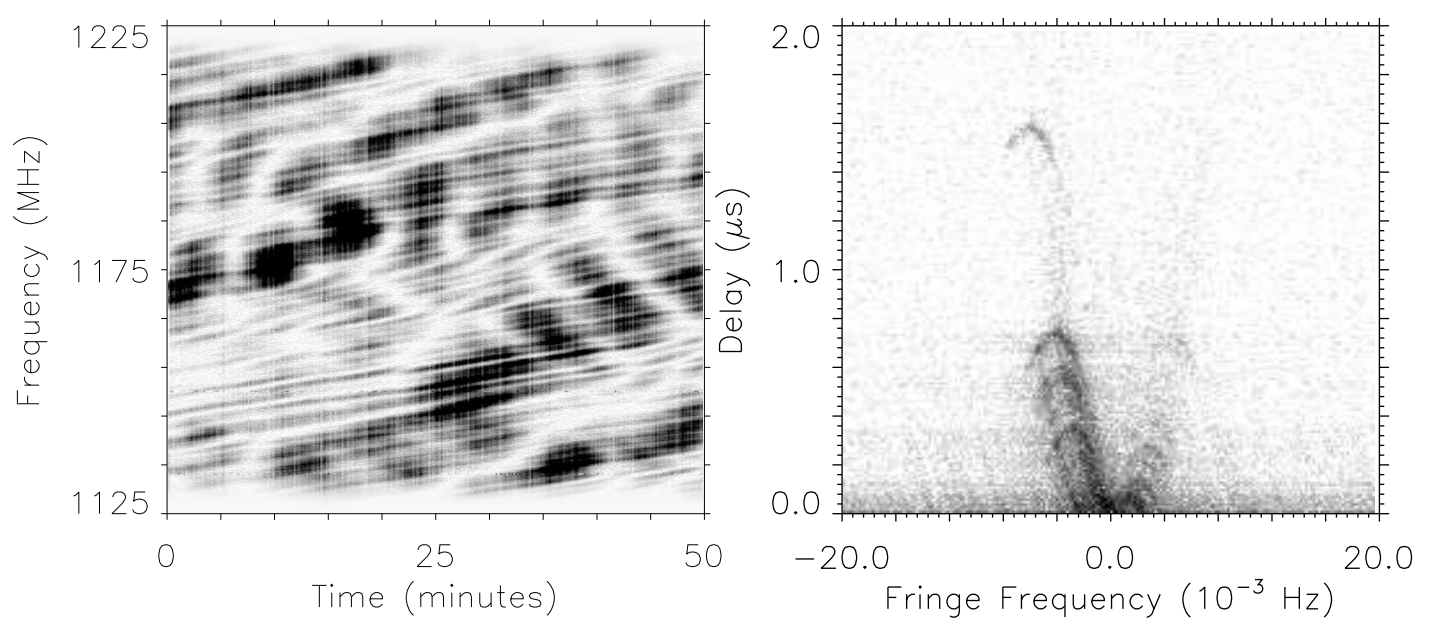

Figure 3. (Left) A dynamic spectrum of PSR B0834+06 observed at Arecibo. Flux density is indicated using a linear grayscale with dark representing the strongest power. The vertical striping is caused by a small number of pulses per $10 \mathrm{~s}$ resolution element. The criss-cross pattern is somewhat unusual and is caused by interference effects when flux arrives from a few discrete directions. (Right) The secondary spectrum (2d power spectrum of the dynamic spectrum) of the same data. The overall parabolic (scintillation) arc indicates that the majority of the scattering occurs in a relatively confined portion along the line of sight. The inverted parabolic "arclets" are due to discrete scattered rays that interfere strongly with the central part of the pulsar image. Power is represented using a logarithmic grayscale (darkest is the strongest power), and the value along the ordinate indicates the overall delay of the scattered ray relative to the central one. From [6].

and analysis of effects, and we use this approximation below, although the apparently common case of multiple screens along the LOS [12] has not been properly analyzed. Following [16] and many other studies we indicate the main scattering angle in the screen as $\theta_{d}$ since it results from diffraction from small scale $\left(10^{5} \mathrm{~m}<l_{d}<10^{9} \mathrm{~m}\right)$ irregularities. All quantities are approximate; see [17] for exact definitions. At an effective distance $D_{\text {eff }}$ from the scattering screen [5, 16] this represents a patch size often referred to as the refractive scale $l_{r} \approx \theta_{d} D_{\text {eff }}$ since irregularities in the screen of this size will act to refract the entire ray bundle [18, 19]. As viewed from Earth, the amount by which the centroid of the ray bundle is refracted is denoted as $\theta_{r}$. There is no guarantee that the ray bundle picture is appropriate for all cases since it is known from spectral studies that pulsars occasionally undergo episodes of multi-imaging in which the image, if it could be resolved, breaks into multiple pieces [6, 16, 20, 21, 22, 23, 24, 25, 26].

For further study of scintillation in the ionized interstellar medium see [17] for a more formal and definitive treatment; 27] for another excellent, physics-oriented approach; good introductory discussions in [1, 28]; and an overview of interstellar turbulence in [29, 30]. 


\section{Correcting for Dispersion Measure Variations}

It is clear from (11) that uncorrected or improperly corrected DM variations will seriously bias GW detection efforts. In an important recent study, Keith et al. [3], building on previous work [31], show that some algorithms for dispersion measure correction also remove gravitational wave power from the signal. As part of the PPTA effort they employ a technique that includes a common mode term in addition to the standard $\nu^{-2}$ term [3]:

$$
t_{\mathrm{OBS}}=t_{\mathrm{CM}}+t_{\mathrm{DM}}\left(\lambda / \lambda_{\mathrm{REF}}\right)^{2}
$$

where $t_{\mathrm{CM}}$ is the wavelength-independent (common mode) delay, $t_{\mathrm{DM}}$ is the dispersive delay at some reference wavelength $\lambda_{\mathrm{REF}}$, and all quantities represent residuals after a basic timing solution has been subtracted. They show that not including the common mode term will result in a bias in the determined dispersion measure. Much more importantly, that bias arises because a portion of the common mode (e.g. an achromatic GW signal) will be absorbed into the chromatic fit for DM.

Keith et al. [3] go on to explore this using a series of simulations that are increasingly realistic. Results of one simulation are shown in figure 4 in which they do not include a common mode term in the fit. In the upper two panels they include gravitational waves $(\mathrm{GW})$ in the stochastic signal present in the realizations. The dashed line, which shows the post-fit power spectrum over 1000 realizations, is strongly suppressed at low frequencies because achromatic gravitational wave power has been absorbed in the chromatic fit for $\operatorname{DM}(\mathrm{t})$. In a comparison (Figure 3 of [3]) they show that this suppression of the gravitational wave signal does not occur when a common mode term is included in the correction. An inevitable consequence of including the common mode term is that a portion of the white noise present in the data ends up in the post-fit residuals. This is a consequence of using the least-squares estimator, which is the minimum variance unbiased estimator.

The NANOGrav collaboration accounts for the covariance between dispersion measure variation and achromatic red noise at a different stage in their analysis pipeline. In [2], they employed a DM correction scheme (called DMX in the TEMPO timing package) that introduces $N_{\text {epoch }}$ independent DM values $\left(D M_{i}\right)$ for that many (nearly simultaneous) dual-wavelength observations. In the pulse time series analysis, the $D M_{i}$ values are fit simultaneously with other pulse timing model parameters (rotation rate, spin-down, position, etc.). The timing analysis package iteratively calculates barycentercorrected TOAs, which serve the same function as common mode ("achromatic") terms. In [2], the effect of the $D M_{i}$ fits on the measurement of a gravitational wave limit was accounted for by considering covariance between the basis functions of the $D M_{i}$ parameters in the timing solution (along with all other timing fit parameters) and putative achromatic gravitational wave signals. More details of this method of accounting for inclusion of DMX parameters are given in [32, 33]; also, see the papers by J. A. Ellis and X. Siements et al. in this volume.

The European Pulsar Timing Array (EPTA) is actively developing a DM correction 


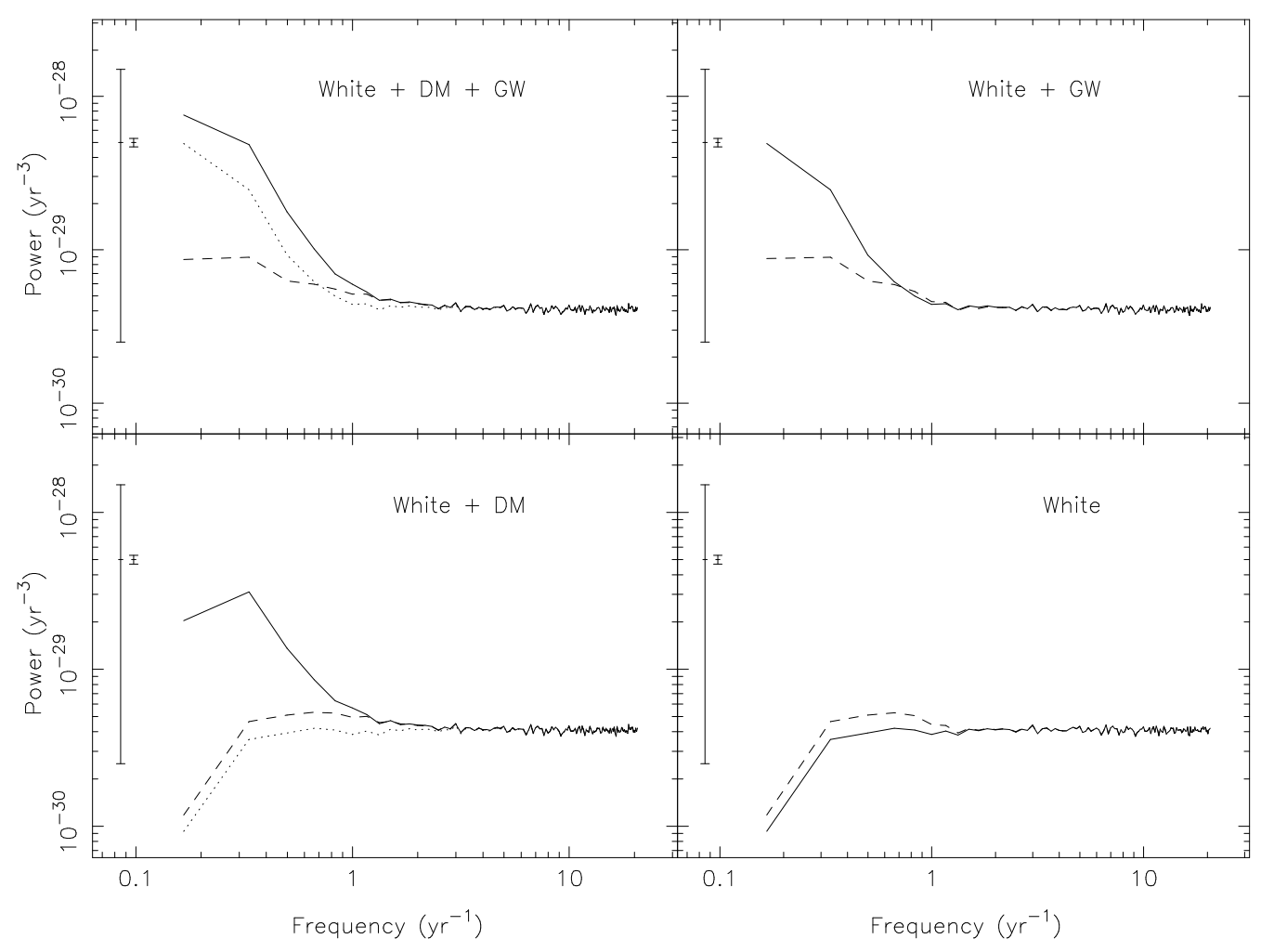

Figure 4. Average power spectra of pre- and post-correction timing residuals with four combinations of signals. The solid line shows the pre-correction spectrum and the dashed line shows the post-correction spectrum. For the two cases in which DM fluctuations are introduced, the dotted line indicates the pre-correction power level without DM fluctuations. The fitting routine uses the $\mathrm{DM}(t)$ interpolated fitting routine, without fitting a common-mode signal. See Keith et al. [3] for further details and a comparison with a trial in which a common mode signal is present in the fit.

scheme as well. Some details were presented at the IPTA meeting in Krabi, Thailand in June 2013, and a full exposition will appear shortly with K. J. Lee as lead author. In broad outline, they develop a new method inspired by the maximum likelihood estimator. Compared to other approaches, their technique utilizes the information that the dispersion measure variation is temporally correlated among adjacent observations. There are two major steps in their method: (i) they measure the power-law spectrum of dispersion measure using a time-domain spectral analysis, and then (ii) they construct the linear optimal

filter to extract its waveform. According to them, this two-step method retains good time resolution to short timescale dispersion variation compared to a polynomial fitting algorithm and is able to handle (inevitable) irregularly sampled data without interpolation because all the signal analyses are performed in the time domain.

Wideband Observing Considerations As detailed elsewhere in this volume, each PTA has its own particular resources and limitations. Members of the NANOGrav team 
have led the development of two wideband spectrometers, PUPPI and GUPPI, sited at Arecibo and Green Bank, respectively. These spectrometers, based on CASPER and compute cluster design, can coherently dedisperse a signal over $800 \mathrm{MHz}$ bandwidth, are extremely versatile, and have become the production spectrometers for the collaboration. Their use raises important new questions for how best to determine arrival times as is discussed by Lommen and Demorest in this volume. The advent of large fractional bandwidths at frequencies of $1 \mathrm{GHz}$ and above raises new opportunities and challenges for DM determination (and higher order ISM effects) as can be seen in figure 5, which is an overlay of observations taken at three different bands with the PUPPI/GUPPI instantaneous bandwidth highlighted. Although each $\sim 1.5 \mathrm{MHz}$ frequency slice has

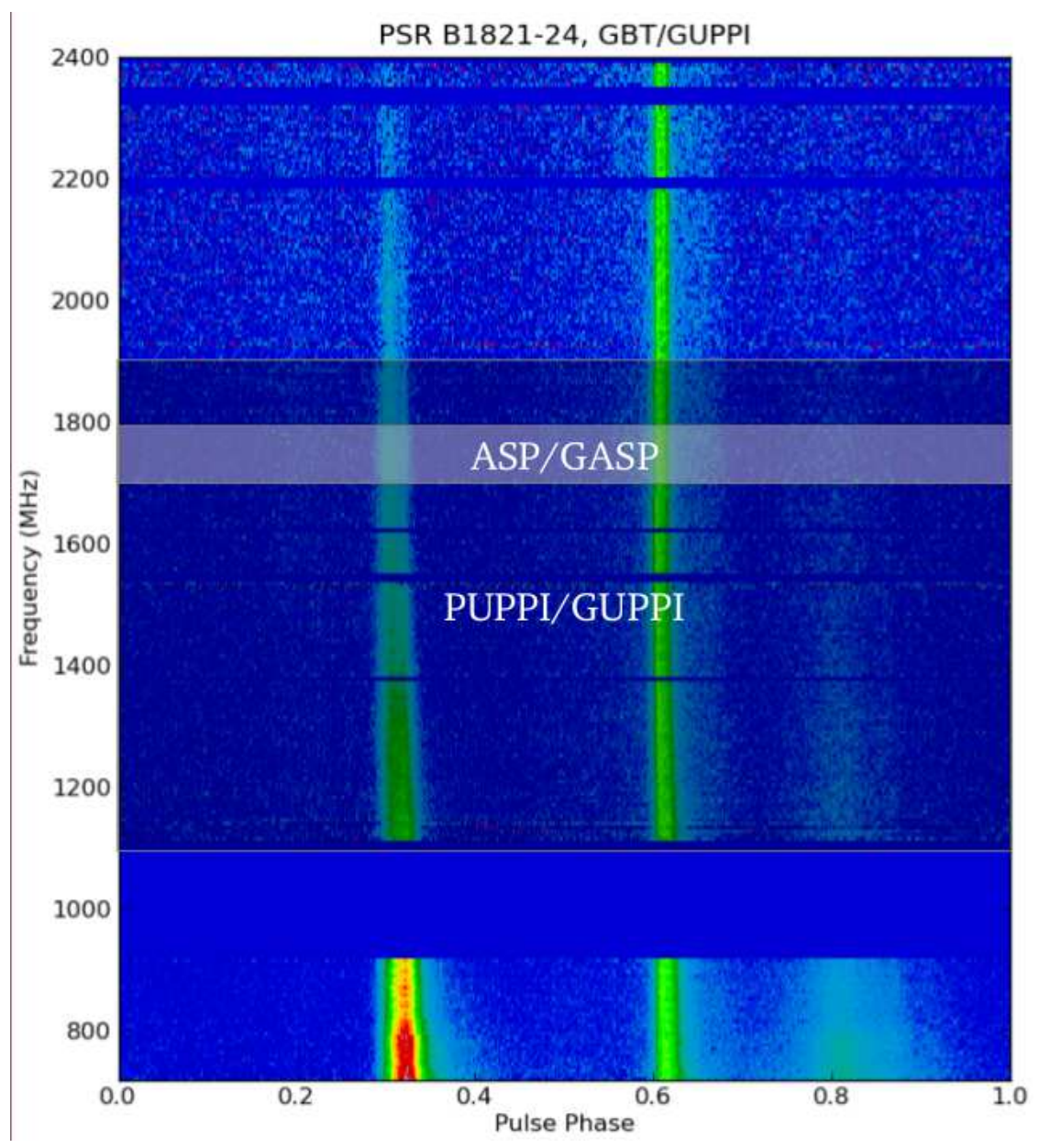

Figure 5. A composite of observations of the globular cluster pulsar M28A taken at three bands with the GUPPI wideband spectrometer. The bandwidth of the previous generation spectrometers (ASP/GASP, $64 \mathrm{MHz}$ ) is shown in comparison to the PUPPI/GUPPI instruments $(800 \mathrm{MHz})$. (The narrow horizontal stripes are due to radio frequency interference excision, and the gap in data between $950-1100 \mathrm{MHz}$ is due to receiver coverage limitations.) Around $800 \mathrm{MHz}$ the pulsar profile clearly becomes triple, and the increased scattering delay at low frequencies becomes apparent. Figure credit: Paul Demorest and Scott Ransom. 
been coherently dedispersed using the same DM value, the extra time delay due to multipath scattering becomes evident at the low frequencies, and even the assumption that one DM value applies over the entire observed range has to be questioned as discussed briefly in section 4. These issues and the obvious profile evolution as a function of time are being explored as central R\&D questions within the various PTAs, which are also moving to wideband observing strategies.

\section{Correcting for Multi-path Scattering Effects}

Many of the ISM scattering delays were identified by Foster and Backer [34]. We follow their framework here, updated and with notation consistent with Cordes and Shanon [16], who identify 6 interstellar scintillation terms:

$$
\Delta t_{\mathrm{ISS}}=\Delta t_{\mathrm{PBF}}+\Delta t_{\mathrm{AOA}}+\Delta t_{\mathrm{AOA}, \mathrm{SSBC}}+\Delta t_{\delta \mathrm{PBF}, \mathrm{RISS}}+\Delta t_{\delta \mathrm{PBF}}+\Delta t_{\mathrm{DM}, \nu}
$$

All of these quantities are time variable, which is the crux of the problem. The first of these, $\Delta t_{\mathrm{PBF}}$, are fluctuations associated with the pulse broadening function (PBF), which results from multi-path scattering. We will loosely refer to the multiple ray paths as a ray bundle, although a full wave propagation formalism [35, 36] is best employed in simulations of the propagation. The image on the sky is distorted and wanders, which gives rise to time variable angle-of-arrival (geometric) delays $\Delta t_{\mathrm{AOA}}$. The changing location of the effective position of the source then gives rise to an additional error when the TOA is improperly corrected to the solar system barycenter, $\Delta t_{\mathrm{AOA}, \mathrm{SSBC}}$. Diffractive and refractive modulation of the propagation alter the PBF and are denoted as $\Delta t_{\delta \mathrm{PBF}}$ and $\Delta t_{\delta \mathrm{PBF}, \mathrm{RISS}}$, respectively. Finally, the ray bundle itself is frequency dependent, and this is encapsulated in $\Delta t_{\mathrm{DM}, \nu}$. Using an empirical model of scattering as a function of

DM, Cordes and Shannon [16] plot scattering time as a function of observing frequency and DM in Figure 6.

Following [16] we elaborate on each of these effects below.

(i) Long-term pulse broadening function fluctuations $\Delta t_{\mathrm{PBF}}$. Assuming a single ray bundle, the geometric delay due to rays at the edge of the bundle relative to the center is $\bar{\tau}_{d}=D_{\text {eff }} \theta_{d}^{2} / 2 c$. If the scattering material has a power spectrum of fluctuations that is nearly Kolmogorov in character [16, 17], and scattering takes place in a thin screen, then the PBF will be well approximated by $p_{d}(t)=$ $\bar{p}_{d}(t) \approx p_{0} \exp -t / \bar{\tau}_{d}$. However, all of these simplifying assumptions are known to be violated at times, and so the PBF may vary substantially in ensemble average from this idealized form for weeks to months. As the large pulsar space velocity carries the LOS through the ISM, $\bar{p}_{d}(t)$ will change slowly with time, $p_{d}(t)=\bar{p}_{d}(t)+\delta p_{d}(t)$. The timescale for change is dictated by the transverse size, $l_{\text {struc }}$, over which the medium changes substantially in scattering properties, magnetic field structure (which affects the anisotropy level of the image [15, 37, 38]), or the propagation crosses filamentary or other discrete structures in the medium and is given by 


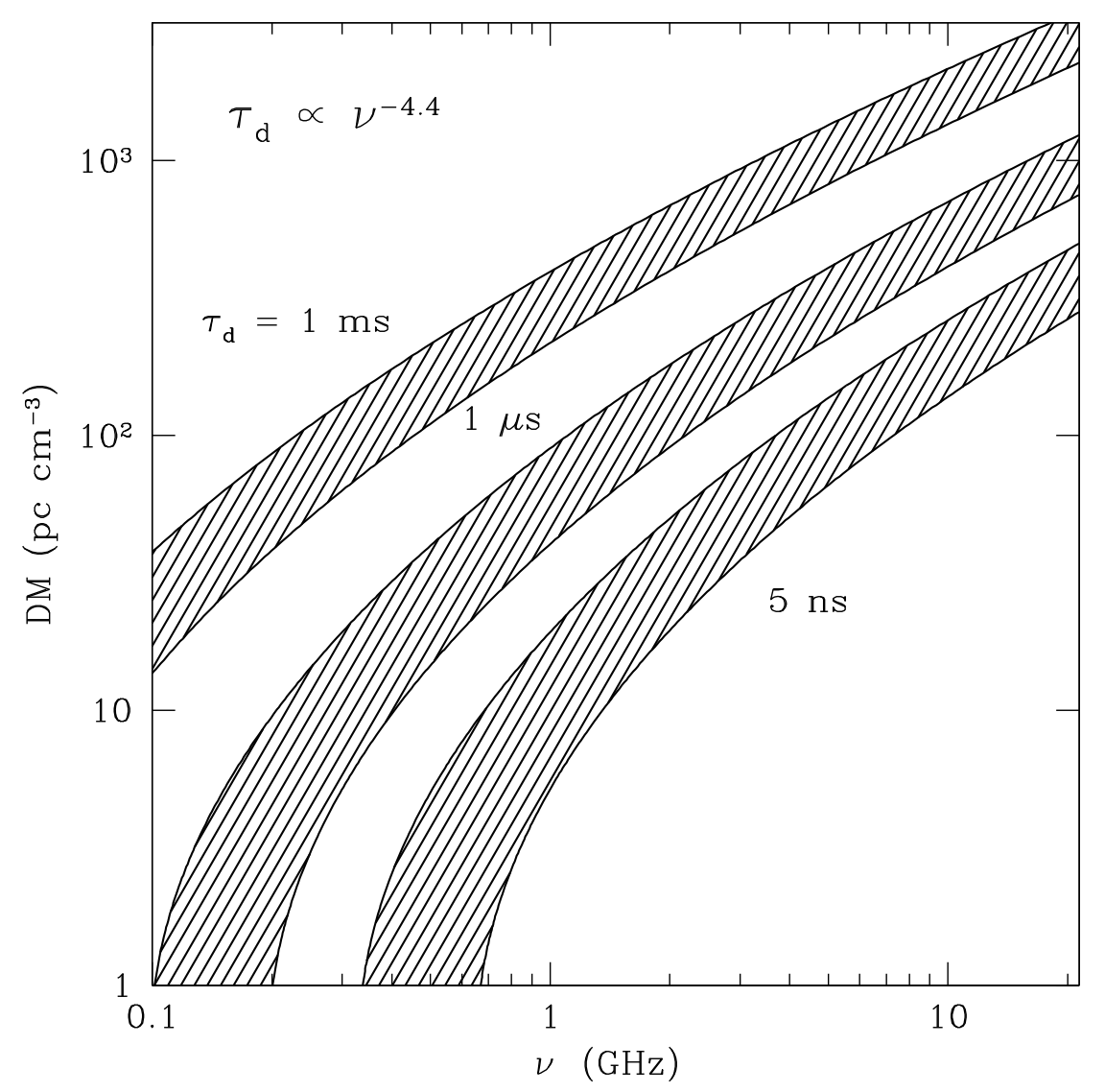

Figure 6. Contours of constant pulse broadening time using an empirical fit to $\tau_{d}$ including $\pm 1.0 \sigma$ deviations from the fit[16]. A $\tau_{d} \propto \nu^{-4.4}$ scaling with frequency is used. This scaling is steeper than appropriate for some high DM objects but is typical for low-DM pulsars like the ones that predominate in the PTA source lists. From [16].

$T_{\text {struc }}=l_{\text {struc }} / V_{\text {eff }}$. The value of $\bar{\tau}_{d}$ can be inferred in many ways, and doing this accurately is one of the main $R \& D$ goals of interstellar mitigation groups since this is the largest contributor to the ISM error budget after dispersion measure variations. At the most basic level we have

$$
2 \pi \bar{\tau}_{d} \Delta \nu_{d}=C_{1},
$$

where $\Delta \nu_{d}$ is the decorrelation (diffractive) bandwidth of the spectrum [16, 39], and $C_{1}$ depends on the medium and its distribution along the LOS [5, 16, 40, but is of order unity. The long-term variation of $\Delta t_{\mathrm{PBF}}$ is not well determined along any line of sight. However, rapid variations in DM (indicating passage through discrete structures) as well as one study of $\Delta t_{\mathrm{PBF}}$ on weeks to months timescale [4] and studies of scattering variability in PSR B1937+21 [41, 42] indicate that $\Delta t_{\mathrm{PBF}} \sim \bar{\tau}_{d}$. 
(ii) Geometrical delay from angle of arrival (AOA) fluctuations $\Delta t_{\mathrm{AOA}}$. Inhomogeneities on the scale of $l_{r}$ cause flux variations, image distortions, and a shift in the centroid of the image [16, 18]. Although scattering in a (reference scenario) of homogeneous, isotropic scattering with a Kolmogorv power spectrum of fluctuations produces $\theta_{r} \lesssim \theta_{d}$, we know from observations that the ISM is this well behaved primarily in the calculations of theorists and the code of modelers! Deviations from this reference scenario are particularly relevant to long duration, high precision PTA observations and are summarized in [15] and references therein. If $\theta_{r}$ is the departure of the AOA from the direct path as viewed by an observer, the induced time delay is

$$
\Delta t_{\mathrm{AOA}} \approx \frac{1}{2 c} D_{\mathrm{eff}} \theta_{r}^{2} \approx 1.21 \mu s D_{\mathrm{eff}}(\mathrm{kpc}) \theta_{\mathrm{r}}^{2}(\mathrm{mas}) .
$$

for a medium at an effective distance $D_{\text {eff }}$ producing a refraction angle of 1 mas [16]. For our reference scenario, $\theta_{r} \lesssim \theta_{d}$, and $\theta_{d}$ can be estimated from equation (4), so that $\Delta t_{\mathrm{AOA}} \sim 160 \mathrm{~ns} \Delta \nu_{d, \mathrm{MHz}}^{-1}$. For typical PTA pulsars, $\Delta \nu_{d, \mathrm{MHz}} \sim 10 \mathrm{MHz}$ at $1 \mathrm{GHz}$, although there is large variation around this value.

(iii) Error in correcting to the solar system barycentre (SSBC) because of angle of arrival fluctuations $\Delta t_{\mathrm{AOA}, \mathrm{SSBC}}$. As discussed in [1], pulse arrival times need to be referred to the solar system barycentre using the exact position of the pulsar. Since AOA fluctuations offset the position, they will drive an error term that, if the position error had no time dependence of its own, would have a one year period with amplitude [16]

$$
\Delta t_{\mathrm{AOA}, \mathrm{SSBC}} \approx 2.4 \mu s \theta_{r}(\mathrm{mas}) \cos \lambda,
$$

where $\lambda$ is the ecliptic latitude of the source. Fluctuations of the AOA on the refractive timescale will modulate the annual periodicity in a stochastic fashion.

(iv) Refractive fluctuations of the $P B F \Delta t_{\delta \mathrm{PBF}, \mathrm{RISS}}$. These are conceptually distinct from the $\Delta t_{\mathrm{PBF}}$ variations, but may be similar in magnitude and difficult to distinguish observationally. As discussed in detail in [16] these are delays connected with the expansion and contraction of the ray bundle by refractive scintillation. The magnitude of this effect is $\Delta t_{\delta \mathrm{PBF}, \mathrm{RISS}} \sim \bar{\tau}_{d}$, but there is disagreement in the literature over whether there is correlation or anti-correlation between $\Delta t_{\delta \mathrm{PBF}, \mathrm{RISS}}$ and $G$, where $G$ is the refractive gain! Cordes and Shannon [16] use a ray optics argument in their Appendix B and elsewhere to find a correlation, $t_{\mathrm{PBF}, \mathrm{RISS}}=G \bar{\tau}_{d}$, for the simplest case of a circular symmetric image $(G=1$ for an unrefracted image) with generalizations to non-symmetric images. However, based on a careful wave propagation simulation, Coles et al. [36] argue that there is an anti-correlation between $G$ and the refractive fluctuations about the mean delay value of $\bar{\tau}_{d}$. There is long-standing discussion of the situation in the literature [43, 44, 45], and it clearly depends (both theoretically and observationally) on how effectively the dominant dispersion delay is removed from the data. Ultimately, we side with the empirical 
observation of an anti-correlation between refractive flux variation and arrival time [36, 45].

(v) Diffractive fluctuations of the $P B F \Delta t_{\delta \mathrm{PBF}}$. The situation with $\Delta t_{\delta \mathrm{PBF}}$ is at least as complicated. Coles et al. [36] state that this situation has not been analyzed theoretically. They argue, however, that there should also be an anti-correlation between flux density $(G)$ and arrival time. When the angular spectrum broadens, more power arrives from larger angles and arrives at a later time. But, more power is scattered out of the beam as well and, hence, the flux density is reduced. This produces the observed anti-correlation between flux and arrival time, operating at the diffractive scale by a different mechanism than at the refractive scale. There is an interesting discussion of $\Delta t_{\delta \mathrm{PBF}}$ in [16] (see, in particular, their Appendix B), but their analytical results must be treated with caution because of the presence of effects with competing signs. Fluctuations of the PBF on both the refractive and diffractive timescale are visible in figure 7.

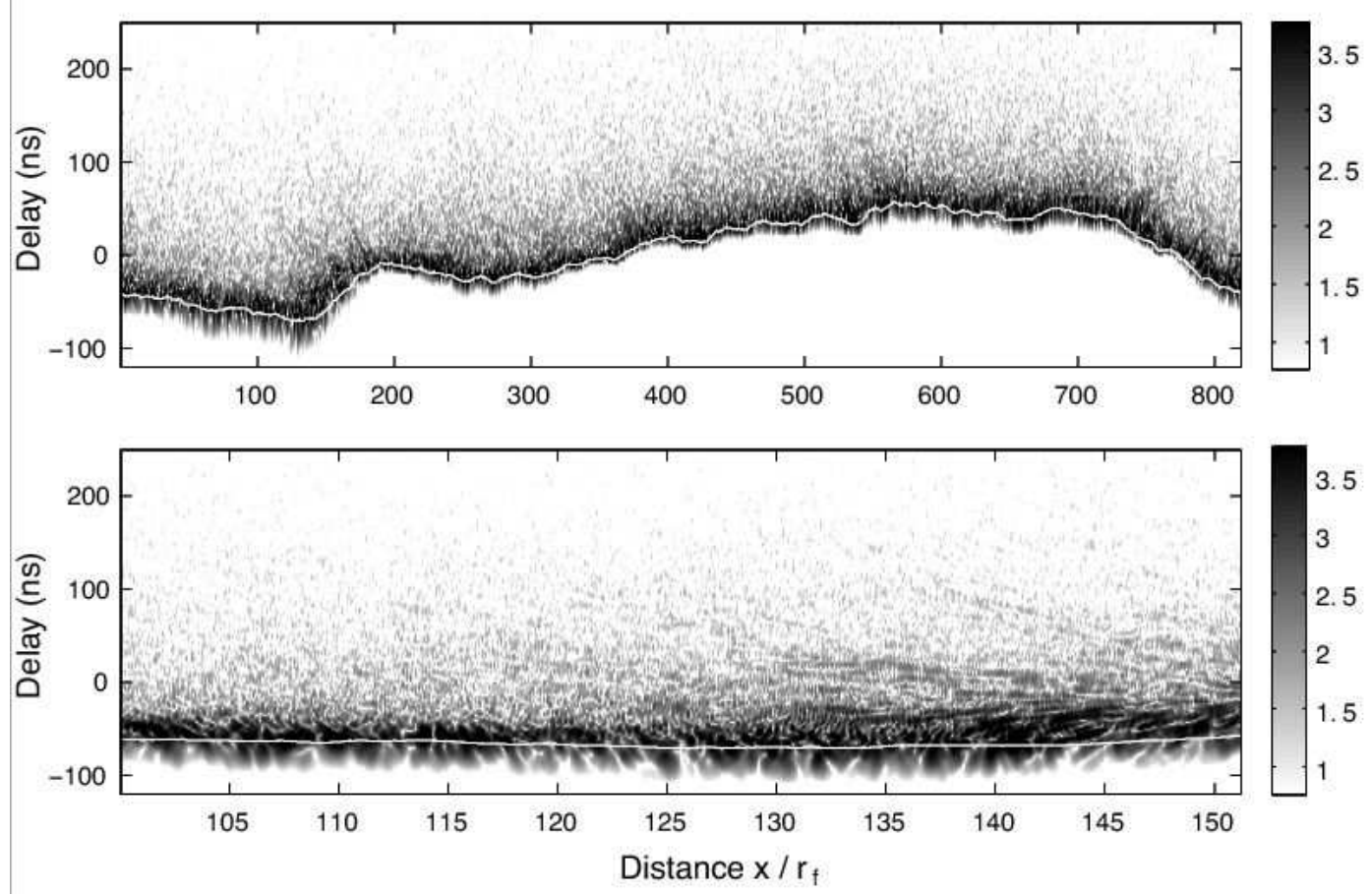

Figure 7. Pulse shape vs position for a simulation of a thin phase-changing screen with Kolmogorov density fluctuations 36. The intensity is $\log 10$ (power). The lower panel is an expanded section of the upper one. In units of the Fresnel scale the diffractive scale is $s_{d}=0.2 r_{f}$ and the refractive scale is $s_{r}=5 r_{f}$ for this simulation. The white line is the group delay through the screen at the same transverse location as the intensity measurement. At a transverse speed of $100 \mathrm{~km} / \mathrm{s}$ the entire simulation represents a duration of about 30 days (see the original paper for more details).

(vi) Frequency dependent variations of dispersion measure $\Delta t_{\mathrm{DM}, \nu}$. In a homogeneous medium there is only one value of the dispersion measure. Multi-path scattering 
changes that, however, because the volume over which DM is evaluated will be frequency-dependent, growing rapidly toward lower frequencies. In the thin screen approximation, and assuming $\theta_{\text {scatt }} \propto \nu^{-2}$, the area contributing to the DM in the screen will be $A_{\text {scatt }} \propto \nu^{-4}$. This gives rise to another source of timing fluctuation, which Cordes and Shannon [16] estimate to have an rms of

$$
\Delta t_{\mathrm{DM}, \nu} \approx 0.12 \mu s D^{5 / 6} \nu^{-23 / 6}\left(\frac{\mathrm{SM}}{\mathrm{kpc} \mathrm{m}^{-20 / 3}}\right),
$$

where $D$ is the screen-Earth distance (in $\mathrm{kpc}$ ), $\nu$ is expressed in $\mathrm{GHz}$, and the scattering measure, which is the path integral of the magnitude of the electron density power spectrum [46], has a value of $\mathrm{SM} \approx 10^{-3.5} \mathrm{kpc} \mathrm{m}^{-20 / 3}$ for nearby pulsars, although much larger values are possible if the LOS intercepts a region of enhanced scattering.

\section{Phase Reconstruction Techniques and Cyclic Spectroscopy}

The introduction of coherent dedispersion by Hankins [47] recognized the fact that dispersion is an electromagnetic phase wrapping characterized by one number (the DM) and can be completely removed through the application of the exact inverse filter operating on a pre-detection (i.e. proportional to the $E$-field) version of the signal. The development of increasingly powerful signal processors has broadened the applicability of the technique, and it has become ubiquitous in high precision pulsar timine. For nearly a decade Walker and a few others have pursued the generalization of this analysis: a coherent descattering of the signal to completely remove the effects of multi-path scattering [48, 49]. This work had been partially successful, although the need to proceed iteratively without direct access to the e-m phase hampered its applicability.

In 2009 Demorest introduced a new approach to the problem based on a signal analysis technique known as cyclic spectroscopy (CS), which was developed by mechanical engineers studying rotating machinery, but not recognized as important in

radio astronomy (see references to the engineering literature in [50]). As the name implies the technique is only applicable to what are known as cyclostationary signals; fortunately, pulsar signals fall into this category. What follows is a very brief sketch of the basics. See [50] or the forthcoming [51] for more details.

Let $X(\nu)$ be the frequency domain representation of a pulsar signal. Then $S_{x}\left(\nu ; \alpha_{k}\right)$ is the cyclic spectrum of $X(\nu)$ where

$$
S_{x}\left(\nu ; \alpha_{k}\right)=E\left\{X\left(\nu+\alpha_{k} / 2\right) X^{*}\left(\nu-\alpha_{k} / 2\right)\right\}
$$

and $\alpha_{k}=k / P$, with $P$ the pulsar period, $k=0,1,2, \ldots$, and the expectation value over many pulses is indicated. If $y(t)$ is the result of passing $x(t)$ through a linear, time-invariant filter with impulse response $h(t)$ (frequency response $H(\nu)$ ),

$$
y(t)=h(t) \star x(t)
$$




$$
Y(\nu)=H(\nu) X(\nu)
$$

then the cyclic spectra of $x$ and $y$ are related by:

$$
S_{y}(\nu ; \alpha)=H\left(\nu+\alpha_{k} / 2\right) H^{*}\left(\nu-\alpha_{k} / 2\right) S_{x}\left(\nu ; \alpha_{k}\right)
$$

If $H(\nu)$ represents the effect of the ISM on the pulsar signal, (11) shows that the phase content of $H(\nu)$ is preserved in the cyclic spectrum, whereas in a standard power spectrum the only information present is the filter magnitude $|H(\nu)|^{2}$. (For simplicity we remove the effect of dispersion from this linear filtering process and deal with it separately in a coherent dedispersion step, so $H(\nu)$ represents the remaining ISM effects.) This can also be understood in terms of the convolution theorem. Pulsars, like other radio sources, emit uncorrelated broadband noise. Modulation of the noise process by the pulsar beaming is a multiplicative operation in the time domain. The counterpart of this is convolution in the frequency domain. Convolution introduces correlation into the phase structure of the signal. Fourier complementarity means that a pulse width of $W$ results in a correlation of the phase over a frequency interval $\sim 1 / W$. This correlated phase (within one pulse) is retained when it passes through $H(\nu)$, and the cyclic spectrum makes the phase of $H(\nu)$ recoverable through the conjugate, shift, and multiply nature of (11). Exactly how to recover $\Phi_{H}$ from (11) is a substantial subject in itself and is the focus of the forthcoming paper [51]. Once the amplitude and phase of $H(\nu)$ have been recovered, a coherent descattering of the signal is straightforward in principle.

The above basics represent the more ambitious use of CS in pulsar signal processing, but CS has an important advantage over standard techniques even if coherent descattering is not the goal. This is illustrated graphically in figure 8 [50. This shows a dynamic spectrum produced with CS (left) and traditional techniques (right). By coherently using a much longer data span than is possible with standard techniques, CS can achieve a much finer time and frequency resolution, which can be especially valuable when observing millisecond pulsars with substantial scattering, hence values of $\Delta \nu_{d} \ll 1 \mathrm{MHz}$. Recently Jones in the NANOGrav collaboration has developed a real-time CS capability based on similar reconfigurable digital hardware as PUPPI and GUPPI, which is available at Arecibo and Green Bank [52]. This is being put into routine operation for supplemental data-taking in parallel for each of our standard timing runs.

\section{Mitigation Techniques}

The comprehensive arrival time model presented in [16] can be expressed as

$$
t_{\nu}=t_{\infty}+t_{\mathrm{DM}}(\nu)+t_{C}(\nu)+t_{\mathrm{W}}(\nu),
$$

where $t_{\infty}$ is the arrival time at infinite frequency, and the other terms represent, respectively, DM fluctuations, other chromatic (primarily scattering related) terms, and a white noise contribution made up of radiometer noise and pulse jitter noise due to 

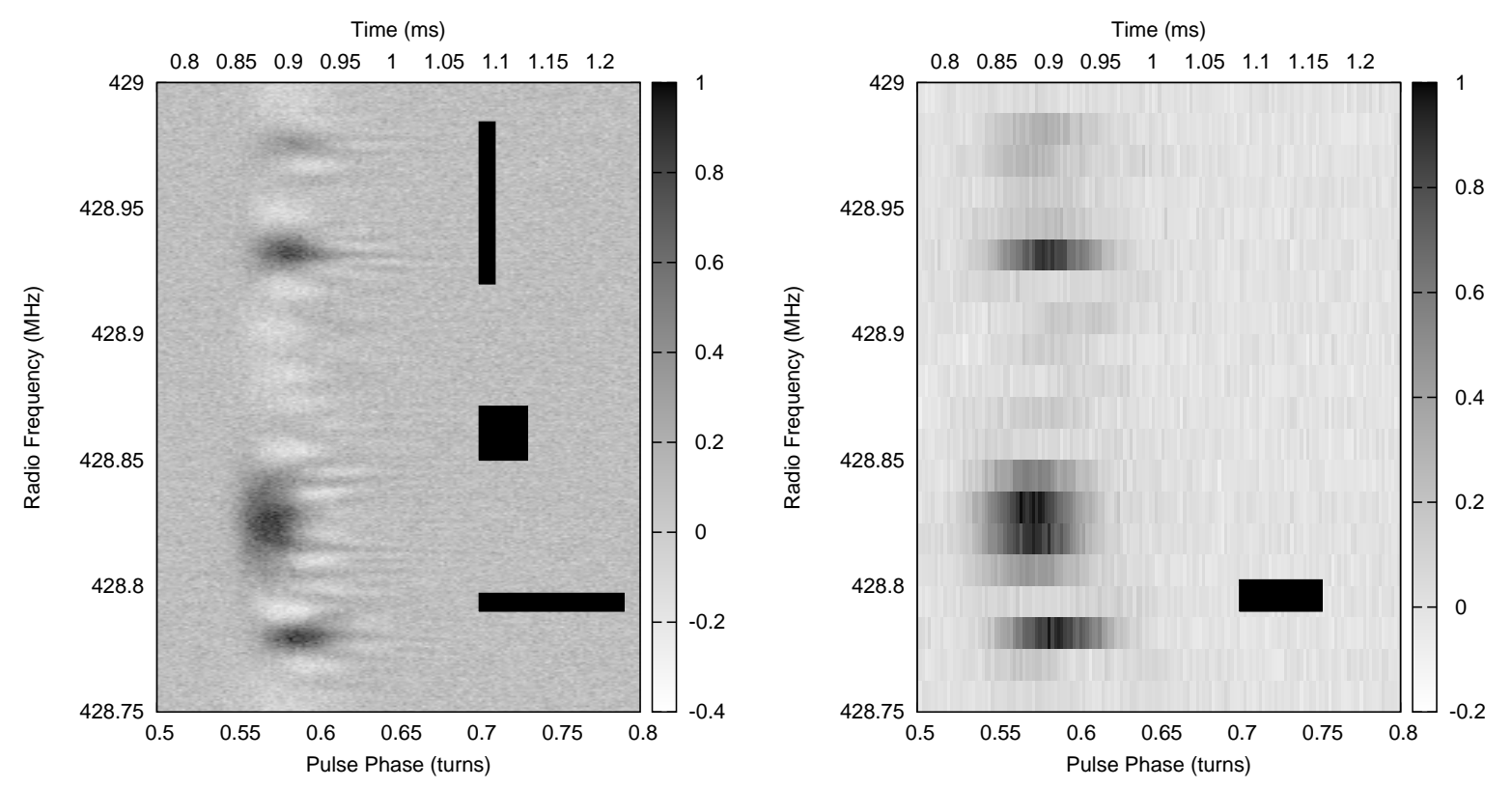

Figure 8. From Demorest 2011 [50]. Left: Detail of periodic spectrum of B1937+21. The black rectangles each contain unity time-frequency area. Unlike a traditional dynamic spectrum the periodic spectrum incorporates e-m phase information resulting in positive(dark) and negative (light) regions on fine scales. See the original article for details. Right: The same data processed with a standard coherently dedispersed filter bank using a frequency resolution of $12.5 \mathrm{kHz}$ and corresponding intrinsic time resolution of $80 \mu \mathrm{s}$ (black rectangle). See [50] for more details.

the finite number of pulses included in any observation. Cordes and Shannon consider two broad approaches to the mitigation of ISM effects: i) correct for DM variations, but ignore refractive and multi-path scattering effects and ii) use multi-wavelength (and broadband) information to aggressively remove all chromatic effects. They lay out a detailed plan of attack in both approaches, but the test of strategy against empirical performance is in its infancy. We refer the reader to [16] as an excellent starting point in developing a comprehensive mitigation strategy and encourage the PTAs to intercompare results in this important arena.

\section{Future Developments}

Very few of the areas outlined above are settled at the level of precision needed for successful PTA timing efforts, particularly when we consider the multi-year to multidecade duration of those observations. R\&D will continue in all of these areas with various PTAs taking the lead on issues of particular importance to them and the IPTA providing structure to sort out optimal solutions.

PTA-based science produces numerous ancillary projects of great interest to communities outside of gravitational studies. The data we are taking, in both its precision and scope, will be of enormous benefit to the study of the ionized interstellar 
medium on a large range of size scales. Subgroups within the three PTA collaborations pursue this ancillary science, and we can expect many exciting results over the next decade.

Looking ahead on a decade or longer timescale, several researchers have highlighted the enormous benefits (particularly in terms of greatly improved angular resolution in the localization of a GW source) to accurately determining the length of one or more "arms" of the Galactic scale interferometer the IPTA is constructing [53]. The requisite accuracy is a fraction of the gravitational wavelength involved, so we might set \pm 1 ly as a reference goal. Recent high-precision VLBI work [54, 55] is approaching this goal for some pulsars, and this is likely to serve as an important element in bootstrapping to the needed precision. Building on ideas and results of the last several decades [6, 7, 8, 10, 12, 56, Pen (CITA) has been championing the use of interstellar scattering as a powerful tool to determine pulsar distances to the needed accuracy, particularly by including the use of VLBI techniques [57].

Using a single-dish example we underscore the potential of high precision astrometry employing multi-path scattering from AU-sized structures in the ISM. In figure 9 we show that the scintillation arc(s) for a nearby, lightly scattered pulsar can be remarkably sharp. Although this has not been fully explored yet for the millisecond pulsars in typical PTAs, there is no doubt that some pulsars in the sample - perhaps even particularly important ones from a timing precision/sensitiviy point of view - will have equally sharp arcs because of scattering over a very small range of the LOS to the pulsar. The curvature of a scintillation arc is a sensitive function of the relative velocities involved [6]. When the Earth's motion is large compared to the pulsar's motion, it is possible to see the effect of that motion as a function of time of year. This is shown in the right hand panel of figure 9 [58].

Overlaid on the data points are three curves for the location of the scattering screen as a function of the fractional distance from the pulsar to the Earth. In this case we were able to determine the fractional distance to $\pm 1 \%$. Although this is neither the required quantity (distance to the pulsar), nor the requisite precision, nor an absolute (versus a relative) measurement, this and other work [38] hints at the enormous potential for ultrahigh precision astrometry using pulsar scintillation with large telescopes, particularly when linked using VLBI techniques.

\section{Summary}

Based on the discussion above and similar research we emphasize the following points:

(i) Individual lines of sight to important PTA pulsars must be monitored and mitigation strategies tailored to the ISM conditions along that LOS. Multiwavelength and broadband techniques are crucial to this effort.

(ii) Accurate and careful correction for dispersion measure variations is an essential component to successful PTA timing efforts and is not yet a settled topic despite important recent contributions. 

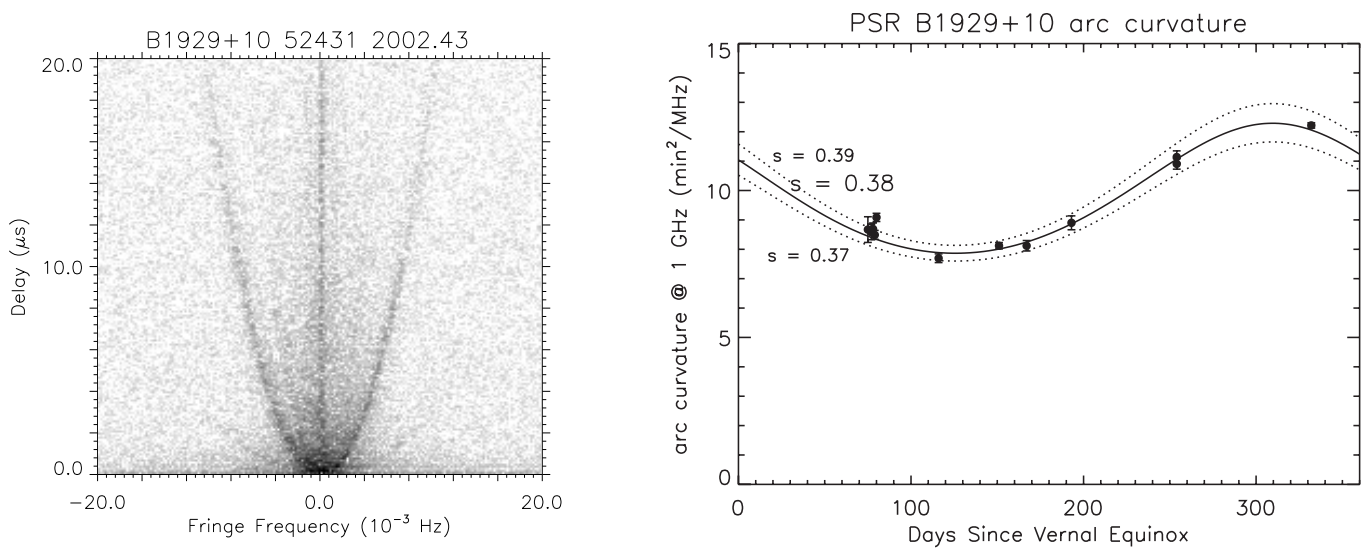

Figure 9. (Left) A secondary spectrum for B1929+10 obtained in a one hour observation with the Arecibo telescope [6]. The sharply delineated parabolic (scintillation) arc is due to scattering in a highly localized screen along the LOS and also requires an anisotropically scattered image [6, 15. Inspection of this and secondary spectra from other epochs shows the presence of two other scintillation arcs, which vary in prominence over multiple years [12. (Right) An analysis of scintillation arcs like the one on the left, along with estimates of the pulsar distance and its measured proper motion, allow the scattering screen to be located, expressed here as a fraction of the distance from the pulsar to the Earth. See [6] for details and caveats. This figure originally appeared in [58.

(iii) Multi-path scattering introduces a myriad of effects at the several microsecond level and below. These need to be determined and mitigated through the use of multi-frequency or ultra-broadband observations and effective algorithms.

(iv) Cyclic spectroscopy and other phase retrieval techniques are likely to play an increasingly important role in interstellar mitigation efforts.

(v) Pulsar scintillation may provide a crucial tool in determining high precision distances to pulsars, greatly enhancing the characteristics of PTAs as gravitational wave detectors.

\section{Acknowledgments}

We thank the members of the NANOGrav interstellar medium mitigation (IMM) working group for their comments and contributions as well as important suggestions from NANOGrav members Justin Ellis, Joseph Lazio, and David Nice. We gratefully acknowledge the support of the NSF through AAG grant 1009580 and PIRE award number 0968126. Comments from both referees were valuable in improving the manuscript. 


\section{References}

[1] Lorimer D R and Kramer M 2005 Handbook of Pulsar Astronomy (Cambridge University Press)

[2] Demorest P B, Ferdman R D, Gonzalez M E, Nice D, Ransom S, Stairs I H, Arzoumanian Z, Brazier A, Burke-Spolaor S, Chamberlin S J, Cordes J M, Ellis J, Finn L S, Freire P, Giampanis S, Jenet F, Kaspi V M, Lazio J, Lommen A N, McLaughlin M, Palliyaguru N, Perrodin D, Shannon R M, Siemens X, Stinebring D, Swiggum J and Zhu W W 2013 ApJ 76294

[3] Keith M J, Coles W, Shannon R S and et al 2012 MNRAS 4292161

[4] Hemberger D A and Stinebring D R 2008 ApJ 674 L37-L40

[5] Cordes J M and Rickett B J 1998 ApJ 507 846-860

[6] Cordes J M, Rickett B J, Stinebring D R and Coles W A 2006 ApJ $637346-365$

[7] Stinebring D R, McLaughlin M A, Cordes J M, Becker K M, Goodman J E E, Kramer M A, Sheckard J L and Smith C T 2001 ApJ 549 L97-L100

[8] Stinebring D R, Hill A S, McLaughlin M A, Becker K M, Cordes J M and Kramer M 2003 Radio Pulsars (Astronomical Society of the Pacific Conference Series vol 302) ed Bailes M, Nice D J and Thorsett S E p 263

[9] Hill A S, Stinebring D R, Barnor H A, Berwick D E and Webber A B 2003 ApJ 599 457-464

[10] Hill A S, Stinebring D R, Asplund C T, Berwick D E, Everett W B and Hinkel N R $2005 A p J L$ 619 L171-L174

[11] Putney M L, Minter A H, Stinebring D R and Ransom S M 2005 American Astronomical Society Meeting Abstracts (Bulletin of the American Astronomical Society vol 37) p 183.15

[12] Putney M L and Stinebring D R 2006 Chin. J. Atron. Astrophys., Suppl. 26 233-236

[13] Stinebring D 2007 SINS - Small Ionized and Neutral Structures in the Diffuse Interstellar Medium (Astronomical Society of the Pacific Conference Series vol 365) ed Haverkorn M and Goss W M p 254

[14] Heiles C and Stinebring D 2007 SINS - Small Ionized and Neutral Structures in the Diffuse Interstellar Medium (Astronomical Society of the Pacific Conference Series vol 365) ed Haverkorn M and Goss W M p 331

[15] Rickett B, Stinebring D, Coles B and Gao J 2011 American Institute of Physics Conference Series (American Institute of Physics Conference Series vol 1357) ed Burgay M, D'Amico N, Esposito P, Pellizzoni A and Possenti A pp 97-100

[16] Cordes J M and Shannon R M 2010 arXiv:1010.3785

[17] Rickett B J 1990 Ann. Rev. Astr. Ap. 28 561-605

[18] Rickett B J, Coles W A and Bourgois G 1984 A $\& A$ 134 390-395

[19] Stinebring D R, Smirnova T V, Hankins T H, Hovis J, Kaspi V, Kempner J, Meyers E and Nice D J 2000 ApJ 539 300-316

[20] Ewing M S, Batchelor R A, Friefeld R D, Price R M and Staelin D H 1970 ApJL 162 L169

[21] Roberts J A and Ables J G 1982 MNRAS 201 1119-1138

[22] Hewish A, Wolszczan A and Graham D A 1985 MNRAS 213167

[23] Cordes J M and Wolszczan A 1986 ApJ 307 L27-L32

[24] Wolszczan A and Cordes J M 1987 ApJ 320 L35-L39

[25] Rickett B J, Lyne A G and Gupta Y 1997 MNRAS 287 739-752

[26] Lambert H C and Rickett B J 1999 ApJ 517 299-317

[27] Narayan R 1992 Phil. Trans. Roy. Soc. A 341 151-165

[28] Lyne A and Graham-Smith F 2012 Pulsar Astronomy (Cambridge University Press)

[29] Elmegreen B G and Scalo J 2004 ARAA 42 211-273

[30] Scalo J and Elmegreen B G 2004 ARAA 42 275-316

[31] You X P, Hobbs G, Coles W A, Manchester R N, Edwards R, Bailes M, Sarkissian J, Verbiest J P W, van Straten W, Hotan A, Ord S, Jenet F, Bhat N D R and Teoh A 2007 MNRAS 378 493

[32] van Haasteren R and Levin Y 2013 MNRAS 4281147 
[33] Ellis J A, Siemens X and van Haasteren R 2013 ApJ 76963

[34] Foster R S and Backer D C 1990 ApJ 361300

[35] Coles W A, Frehlich R G, Rickett B J and Codona J L 1987 ApJ 315 666-674

[36] Coles W A, Rickett B J, Gao J J, Hobbs G and Verbiest J P W 2010 ApJ 717 1206-1221

[37] Goldreich P and Sridhar S 1995 ApJ 438 763-775

[38] Brisken W F, Macquart J P, Gao J J, Rickett B J, Coles W A, Deller A T, Tingay S J and West C J 2010 ApJ 708 232-243

[39] Cordes J M, Weisberg J M and Boriakoff V 1985 ApJ 288 221-247

[40] Lambert H C and Rickett B J 2000 ApJ 531 883-901

[41] Cordes J M, Wolszczan A, Dewey R J, Blaskiewicz M and Stinebring D R 1990 ApJ 349245

[42] Ramachandran R, Demorest P, Backer D C, Cognard I and Lommen A 2006 ApJ 645 303-313

[43] Blandford R D and Narayan R 1985 MNRAS 213 591-611

[44] Romani R W, Narayan R and Blandford R 1986 MNRAS 220 19-49

[45] Lestrade J, Rickett B J and I C 1998 A $\& A 3341068$

[46] Bhat N D R, Cordes J M, Camilo F, Nice D J and Lorimer D R 2004 ApJ 605 759-783

[47] Hankins T H and Rickett B J 1975 Methods in Computational Physics Volume 14 - Radio Astronomy (New York: Academic Press) pp 55-129

[48] Walker M A and Stinebring D R 2005 MNRAS 362 1279-1285

[49] Walker M A, Koopmans L V E, Stinebring D R and van Straten W 2008 MNRAS 388 1214-1222

[50] Demorest P B 2011 MNRAS 416 2821-2826

[51] Walker M A, Demorest P B and van Straten W 2013 ApJ submitted

[52] Jones G, Demorest P B and van Straten W 2013 in preparation

[53] Boyle L and Pen U L 2012 PhysRevD 86124028

[54] Deller A T, Verbiest J P W, Tingay S J and Bailes M 2008 ApJ 685 L67-L70

[55] Deller A T, Boyles J, Lorimer D R, Kaspi V M, McLaughlin M A, Ransom S, Stairs I H and Stovall K 2013 ApJ $\mathbf{7 7 0} 145$

[56] Walker M A, Melrose D B, Stinebring D R and Zhang C M 2004 MNRAS 354 43-54

[57] Pen U L, Macquart J P, Deller A and Brisken W 2013 ArXiv 1301.7505

[58] Stinebring D R, Hill A S and Ransom S M 2005 Binary Radio Pulsars (Astronomical Society of the Pacific Conference Series vol 328) ed Rasio F A and Stairs I H p 349 